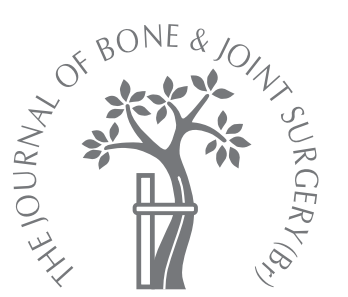

M. J. Challis,

P. Gaston,

K. Wilson,

G. A. Jull,

R. Crawford

From the University

of Queensland and

Queensland

University of

Technology,

Brisbane, Australia

\title{
Cyclic pneumatic soft-tissue compression accelerates the union of distal radial osteotomies in an ovine model
}

The aim of this randomised, controlled in vivo study in an ovine model was to investigate the effect of cylic pneumatic pressure on fracture healing. We performed a transverse osteotomy of the right radius in $\mathbf{3 7}$ sheep. They were randomised to a control group or a treatment group where they received cyclic loading of the osteotomy by the application of a pressure cuff around the muscles of the proximal forelimb. Sheep from both groups were killed at four or six weeks. Radiography, ultrasonography, biomechanical testing and histomorphometry were used to assess the differences between the groups. The area of periosteal callus, peak torsional strength, fracture stiffness, energy absorbed over the first $10^{\circ}$ of torsion and histomorphometric analysis all showed that the osteotomies treated with the cyclic pneumatic pressure at four weeks were not significantly different from the control osteotomies at six weeks.

Many studies have demonstrated the positive effect of cyclic loading on osteogenesis. ${ }^{1-6} \mathrm{We}$ have previously shown in an in vitro ovine model that cyclic loading of a fracture of the distal radius can be achieved with pneumatic soft-tissue compression of the musculature proximal to the fracture. ${ }^{7}$ This was achieved by the rapid inflation and deflation of a cuff. A linear correlation was shown between the pressure generated in the cuff and the compressive force measured at the fracture site.

The aim of this study was to investigate the effect of cyclic loading on fracture healing in an in vitro ovine model. A pilot study showed biomechanical and histological trends towards stronger fracture healing. Our hypothesis was that the application of cyclic loading to fractures of the distal radius would accelerate the rate of healing.

\section{Materials and Methods}

Experimental design. We carried out a transverse osteotomy of the right distal radius on 37 skeletally mature sheep with a mean weight of $35.7 \mathrm{~kg}$ (25.5 to 44$)$. The size of the sample was determined from the results of the pilot study $(n=8)$. A power analysis indicated that nine sheep would be required in each of four groups to show a significant difference at the 0.05 level with 0.8 probability. All sheep were managed post-operatively in a non-weight-bearing splint. Half had cyclic compression, the other half did not receive the treatment. The sheep were killed at either four or six weeks after their osteotomy. In this way, comparisons could be drawn between the two groups at two different time periods. Radiographs, biomechanical testing and histomorphometry were used to determine differences between the two groups. Ultrasonographic measurement of the depth of the foreleg muscles was also conducted to determine whether there were any differences in muscle size between the two groups. Ethical approval for this study was gained from the University of Queensland and the Queensland University of Technology Animal Ethics Committees.

Osteotomy and post-operative management. Anaesthesia was induced with intravenous propofol (Diprivan) 0.5 to $1.0 \mathrm{ml} / \mathrm{kg}$. The animal was then intubated with a 9 to $10 \mathrm{~mm}$ inner diameter cuffed endotracheal tube. The anaesthesia was maintained throughout the surgery with propofol 1 to $2 \mathrm{mls} / \mathrm{kg}$ per hr. All sheep were ventilated. An intra-operative and postoperative analgesic regime was used. Buprenorphine $(0.01 \mathrm{mg} / \mathrm{kg})$ was given intravenously at the time of surgery and another dose intramuscularly at the end of surgery or within eight hours of the initial dose. Further doses were administered every eight hours if required.

Using a lateral approach, a skin incision was made from midway along the length of the radius to $20 \mathrm{~mm}$ proximal to the radiocarpal joint. The incision was continued through the periosteum and onto the bone. The periosteum 


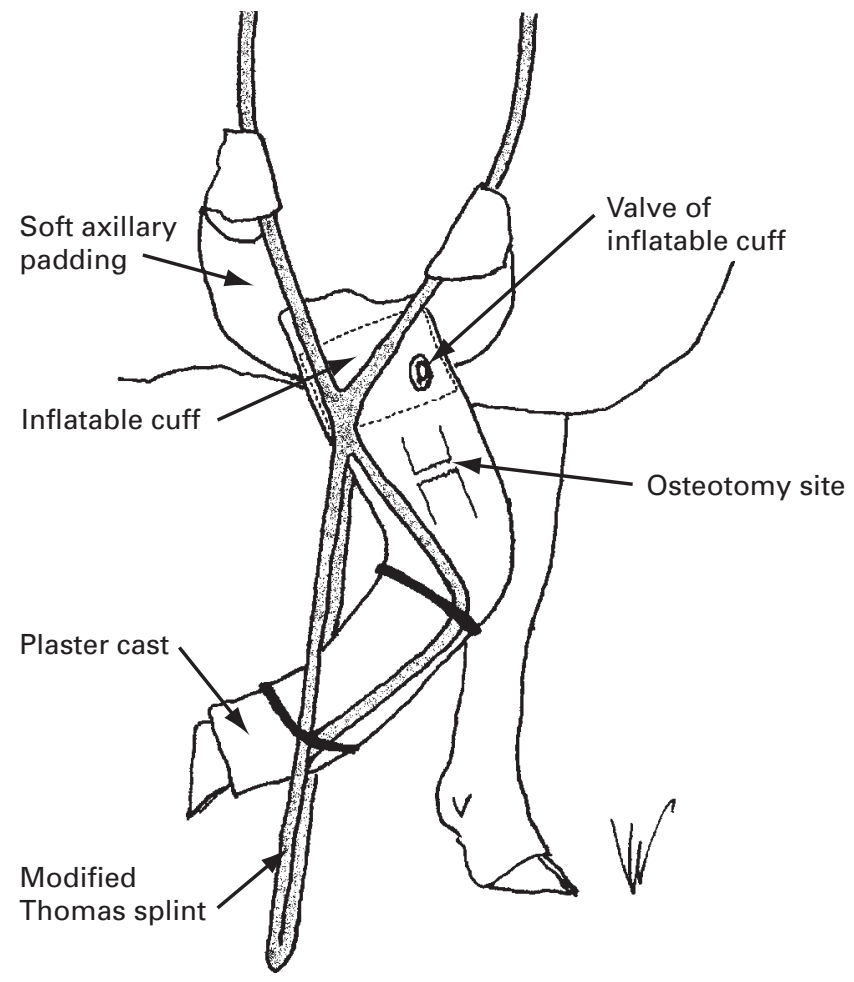

Fig. 1

Diagram of a sheep with a plaster and an external splint to prevent weight-bearing.

was lifted from the bone around its circumference without cutting it further. Two steel spatulas were positioned between the bone and periosteum to protect both the surrounding soft-tissue and the periosteum. The radius was then osteotomised transversely $50 \mathrm{~mm}$ proximal to the radiocarpal joint using a reciprocating saw. Care was taken at this stage to leave the radius intact via a small interconnecting piece of bone. Before the osteotomy was completed, the radius was stabilised using a $110 \mathrm{~mm}$ length of $1.6 \mathrm{~mm}$ diameter Kirschner (K-) wire inserted into the intramedullary canal through a hole drilled obliquely in the distal fragment of the radius, $30 \mathrm{~mm}$ proximal to the radiocarpal joint. With the K-wire in situ, the interconnecting piece of the radius was broken by hand, the incision was closed and the limb enclosed in a plaster cast.

The animals were randomised by having a member of the theatre staff select a sealed envelope from a box. Those sheep that were assigned to the treatment group had an inflatable cuff positioned circumferentially around the musculature proximal to the osteotomy. The cuff measured $400 \mathrm{~mm} \times 100 \mathrm{~mm}$ and was made from a rigid vinyl material. Each cuff had a valve to allow inflation and deflation. To splint the leg, a plaster cast (Vet-Lite, DLC Australia Pty Ltd, Queensland, Australia) was applied with the radiocarpal joint at $60^{\circ}$ of flexion and the metacarpophalangeal joint at $30^{\circ}$ of flexion. A modified Thomas splint was applied externally to the leg immediately following surgery so that the sheep could walk taking weight through the axilla without weight-bearing through the osteotomy (Fig. 1).

Immediately after the operation anteroposterior and lateral radiographs were taken to confirm the osteotomy and the position of the opposing ends. All of the animals were given cephalothin (Mayne Pharma, Melbourne, Australia) intravenously and an intramuscular dose of gentamicin (Mayne Pharma). Sheep were kept in a special recovery pen for one or two days (usually depending on the weather) and then released into a larger enclosure $(25 \mathrm{~m} \mathrm{x} 10 \mathrm{~m})$ where they were managed for the duration of their time in the study.

It had been found in a previous study ${ }^{7}$ that inflating the pneumatic cuff at a certain rate to a particular pressure results in a compressive force at a fracture of the distal radius in an ovine model. The sheep which were assigned to the treatment group began to receive the cyclic-loading treatment via the compression pump apparatus and pneumatic cuff one week after the day of their surgery. The pump apparatus was set to deliver an $8 \mathrm{~N}$ load per cycle to the osteotomy site during the first week of treatment. The load per cycle delivered to the osteotomy site was increased by $2 \mathrm{~N}$ each successive week so that by the fifth week of treatment (sixth week after operation), the load per cycle was $16 \mathrm{~N}$. There were 60 compression cycles per treatment that were delivered in ten minutes. The treatments were given in the morning and then again in the afternoon.

Half of the sheep from each group were killed at four weeks and the rest at six weeks by injection of pentobarbitone sodium (Virbac Animal Health Pty Ltd, New South Wales, Australia; $0.5 \mathrm{ml} / \mathrm{kg}$ ). Both forelimbs were removed at the glenohumeral joint. The intramedullary K-wire was removed from the right leg and radiographs were taken. All specimens were wrapped in saline-soaked cheesecloth and kept on ice until testing was carried out. This was always completed within 24 hours. The depth of muscle tissue was measured using ultrasound before the biomechanical and histological testing.

Ultrasonographic and radiographic evaluation. An ultrasound scan was performed on each limb to determine the depth of muscle tissue between the surface of the radius and the superficial surface of the muscle. The measurements were taken at the same point on each leg, midway between the radial styloid and the tip of the olecranon over the flexor musculature using a $10 \mathrm{~Hz}$ ultrasound transducer (Synergy Diagnostic Ultrasound, software version 3.7 to 3.8, Haifa, Israel). Measurements were taken on both the right and left legs of each animal. The measurement from the right leg was expressed as a percentage of the left leg so that each sheep acted as its own control. Three separate scans and measurements were taken of each leg and the mean of the three measurements was calculated.

Radiographs of each osteotomy were taken at the beginning and end of the intervention period. Several measurements were taken which included the area of periosteal callus and the angulation and translation of the osteotomy site on both the anteroposterior and lateral views. The area 


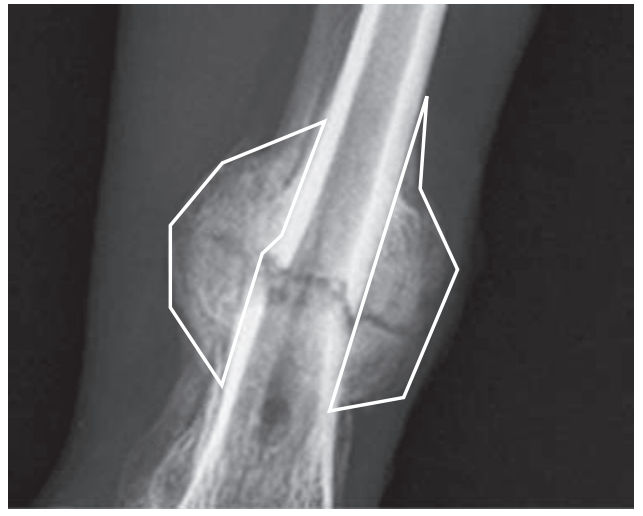

Fig. 2

Radiograph showing how the periosteal callus was outlined. Computer software automatically calculated the area $\left(\mathrm{mm}^{2}\right)$.

of callus was measured using a custom-designed Labview software program (National Instruments Corp., Austin, Texas) that enabled the callus to be outlined with a series of points (Fig. 2). The program calculated the outlined area in square millimetres. Two orthopaedic surgeons (RC, PG) who were blind to the sample examined all the radiographs. When measuring the area of callus, the examiners made a decision about where periosteal callus started and finished. The measurements from the anteroposterior and lateral views of each sheep were combined to create a single measurement for each specimen. The angulation of the fracture was measured using a standard universal goniometer. Translation was measured with a metric ruler.

Biomechanical evaluation. Prior to testing, the soft tissue was removed from the radii. For all the osteotomised radii $25 \mathrm{~mm}$ of tissue was left on either side of the fracture so as not to interfere with the callus. The specimens were mounted in a torsion-testing rig and loaded to failure at an angular displacement rate of $1^{\circ}$ per second. The displacement curve gave a measurement of the peak torque, the fracture stiffness and the energy absorbed. All measurements of the osteotomised radius were normalised against the corresponding values for the contralateral intact radius.

Histological evaluation. After the osteotomised radii were tested to failure in torsion, they were preserved in formalin for histological preparation. A 2 to $3 \mathrm{~mm}$ section through the midsagittal plane was taken from each osteotomised radius using a band saw. This section was chosen because a consistent pattern of periosteal callus formation was seen on the radiographs and this section would show the greatest cross-sectional area of periosteal callus. The sections were kept in decalcifying solution for two weeks. Longitudinal sections of $5 \mu \mathrm{m}$ were cut from the specimens and stained with haematoxylin and eosin for examination using light microscopy.

The histomorphometric characteristics of the fractures were assessed. For each osteotomy, the periosteal callus on the volar side of the fracture was examined. The examiner was blind to the source of the tissue. The histomorphometric analysis calculated the percentage of cartilaginous tissue, woven bone and fibrous tissue. The amount of 'void' space was subtracted from the total area before the percentages of the above tissues were calculated. The 'void' space appeared on the slides because the fractures had been tested to failure in torsion that produced damage to the tissue.

Statistical analysis. The results of the radiographic, biomechanical and histomorphometric measures were analysed using one-way analysis of variance (ANOVA). In these assessments the two four-week groups and two six-week groups were compared. In addition the group that was treated for four weeks was compared with the six-week control group.

A one-way ANOVA was used to compare the amount of muscle wasting in the treated group with that of the control group. The volume of the woven bone from both treated groups was compared with that from both control groups using a $t$-test. A value of $\mathrm{p}=0.01$ was considered significant.

\section{Results}

Two sheep were lost to the trial, one owing to a clostridial infection and the other because of a perianal fistula. The results are presented in Table I.

Ultrasonography. The analysis of variance showed that the pneumatic cuff treatment had no significant effect on muscle wasting. There were no differences between the treated group and the control group measured at four weeks $(\mathrm{p}=0.44)$ and at six weeks $(\mathrm{p}=0.42)$.

Radiography. Prior to the radiographs being examined a reliability study was conducted involving three orthopaedic

Table I. Summary of the results showing the mean of each outcome measure with the $95 \%$ confidence intervals

\begin{tabular}{|c|c|c|c|c|}
\hline Outcome measure & $\begin{array}{l}\text { Four-week treated } \\
\text { group }(\mathbf{n}=8)\end{array}$ & $\begin{array}{l}\text { Four-week control } \\
\text { group }(\mathbf{n}=9)\end{array}$ & $\begin{array}{l}\text { Six-week treated } \\
\text { group }(n=9)\end{array}$ & $\begin{array}{l}\text { Six-week control } \\
\text { group }(n=9)\end{array}$ \\
\hline $\begin{array}{l}\text { Muscle depth (ultrasonography, \% of } \\
\text { contralateral forelimb) }\end{array}$ & 86.7 (84.8 to 88.6$)$ & 84.9 (82.4 to 87.4$)$ & 86.1 (81.8 to 90.4$)$ & 83.5 (78.8 to 88.2$)$ \\
\hline Area of callus on radiograph $\left(\mathrm{mm}^{2}\right)$ & (497 to 831$)$ & $406 \quad(251$ to 561$)$ & (616 to 1140$)$ & (690 to 1110$)$ \\
\hline Peak torque (\% of contralateral forelimb) & (37 to 45$)$ & 28.9 (20.9 to 36.9$)$ & (51.8 to 70.2$)$ & 47.5 (37.5 to 57.7$)$ \\
\hline Stiffness (\% of contralateral forelimb) & 33.9 (27.3 to 40.5$)$ & $17.4(15.8$ to 19.0$)$ & 62.1 (44.9 to 79.3$)$ & $40.7(24.1$ to 57.3$)$ \\
\hline Energy absorbed (\% of contralateral forelimb) & 33.6 (26.7 to 40.5$)$ & $18.4(5.8$ to 31$)$ & 62.7 (43.5 to 81.9$)$ & $40.2(25.3$ to 55.1$)$ \\
\hline Histomorphology ( $\%$ of woven bone in callus) & $32.7(24.7$ to 40.7$)$ & $19.5(12.4$ to 26.6$)$ & $40.5(32.1$ to 48.9$)$ & 29.2 (22.4 to 46.0$)$ \\
\hline
\end{tabular}




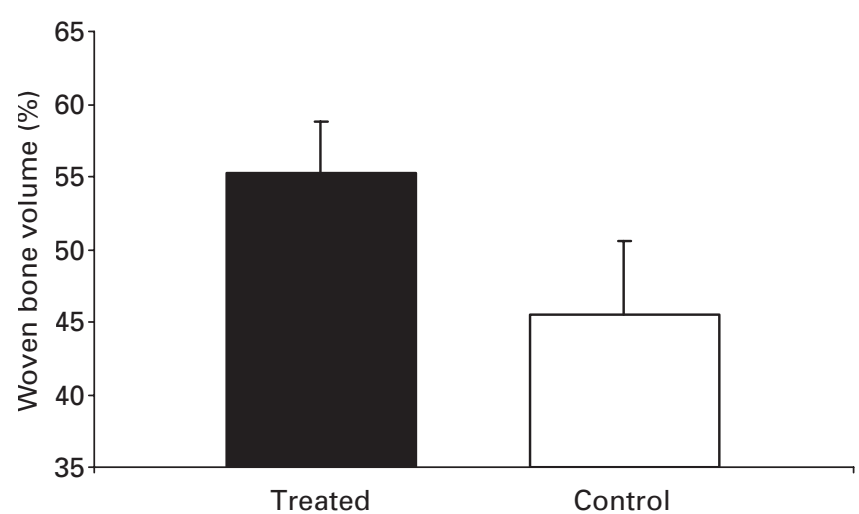

Fig. 3

Graph showing the comparison between the volume of woven bone in the periosteal callus of all the treated fractures and all the control fractures $(p=0.01)$.

surgeons. The results of the area of periosteal callus measured on the anteroposterior and lateral radiographs showed that there was a significant difference between the treated and control groups at four weeks $(\mathrm{p}=0.03)$ but not at six weeks $(\mathrm{p}=0.90)$ (Table I). Notably, the area of periosteal callus in the treated group at four weeks was not significantly different from the control group at six weeks $(\mathrm{p}=$ $0.10)$. There were no significant differences in angulation and translation of the fracture, on anteroposterior and lateral views, between the treated and untreated fractures.

Biomechanical tests. There was a significant difference $(\mathrm{p}=0.006)$ in peak torque, stiffness and energy absorbed between the treated and control groups at four weeks. There was no significant difference $(\mathrm{p}>0.05)$ across all three outcome measures between the treated and control groups at six weeks. The three outcome measures in the treated group at four weeks were no different to the control group at six weeks $(\mathrm{p}>0.05)$.

Histomorphometry. The analysis showed that at both the four- and six-week time period, there were significant differences between the treated and the control groups $(\mathrm{p}=$ $0.03, p=0.04$, respectively). Histomorphometry was also used to calculate the volume of woven bone in the periosteal callus of each osteotomy. This was measured from the midsagittal histological sections of the volar side of each fracture. The data from all the treated sheep were compared with the data from all the control sheep. The results of the analysis (two-tailed $t$-test) showed that there was a significant difference between the volumes of the periosteal woven bone when the treated group was compared with the control group $(\mathrm{p}=0.01)$ (Fig. 3 ).

\section{Discussion}

The design of this study allowed for the direct comparison of healing fractures which had been exposed to a loading regime with controls at four and six weeks. The results of this study concur with other studies that have investigated the effect of applying mechanical loads to healing fractures. Mechanical loading of fractures has been shown to enhance the rate of fracture healing in animal models ${ }^{2,4,8-11}$ and in humans. ${ }^{5,12-15}$ Additionally the initial phase of fracture healing has been shown to be sensitive to mechanical conditions, which subsequently influence the course of healing. ${ }^{16}$ Our study has shown that, in the ovine model, the application of an appropriate mechanical stimulus in the first four weeks has had a significant effect in enhancing the rate of fracture healing.

A notable finding of this study was that, across all measures of fracture healing, the stage of healing at four weeks in the treated group was not significantly different from the control group at six weeks. This was true for the outcome measures; area of periosteal callus from the radiographs $(p=0.1)$, the peak torque, stiffness and the energy absorbed through the first $10^{\circ}$ of torsion for each fracture $(\mathrm{p}=0.26$, $\mathrm{p}=0.47$ and $\mathrm{p}=0.35$, respectively) and the amount of woven bone in the periosteal callus $(\mathrm{p}=0.53)$. Additionally, the volume of woven bone in the callus was significantly greater in the treated groups $(p=0.004)$. Since there was no significant difference between the four-week treated and the six-week control group, the implication is that the fractures of the four-week treated group must have healed under different conditions. The only difference in the management of the two groups was the application of the cyclic loading to the treated group. Therefore, it can be proposed that it was the cyclic loading that was responsible for the faster rate of healing in the four-week treated group.

Both the treated and the control fractures were expected to progress to union. Thus at the six-week end-point it was expected that there would be no significant differences between the treated and control groups. There were however, trends towards evidence of better fracture healing in the six-week treatment group from all aspects of the biomechanical testing. There were also significantly greater ( $p=0.04$ ) amounts of woven bone found in the periosteal callus of the six-week treated sheep when compared with the six-week control group. The lack of difference in the area of callus measured radiographically could suggest that the loading regime administered to the treatment group after four weeks was not optimal. The loading regime increased by a constant amount $(2 \mathrm{~N})$ each week. The progression of a fracture's strength may not be linear during this period and, therefore, it is possible that had the amplitude and duration of the treatment increased at a greater rate after four weeks the differences between the treated and control groups may well have reached significance at six weeks. Further research is required to confirm this hypothesis.

The above argument can be supported from human research. It has been shown in man that fractures of the tibia can safely undertake partial weight-bearing once the torsional strength of the healing fracture has reached 15 $\mathrm{Nm} .{ }^{17}$ The normal torsional strength of the human tibia is $98 \mathrm{Nm} .{ }^{18}$ This indicates that in the fractured human tibia model partial weight-bearing can commence when the strength of the fracture has reached approximately $15 \%$ of 
the normal torsional strength. The treated sheep in the current study reached a mean torsional strength of $41 \%$ by four weeks. These sheep could have commenced weightbearing at four weeks, or even earlier, if the findings for man are extrapolated to sheep. This could have had a beneficial effect on healing of the fracture.

The optimal dosage of cyclic loading is still to be determined. A review of the literature for the dosages used in experimental trials that have produced a significant difference in healing rates provides some direction. The studies of Panjabi et $\mathrm{al}^{2}$ and Wolf et $\mathrm{al}^{19}$ indicate that rather than continuous loading from the first day after injury, rigid immobilisation was necessary during the initial stages of healing. The initial period of immobilisation was followed by the application of cyclic loads which led to enhanced rates of healing. More recent studies have shown that relatively early application of controlled interfragmentary micromovement or dynamisation enhances osteogenesis. ${ }^{20-23}$ Additionally, Kenwright and Gardner, ${ }^{24}$ state that the force of the cyclic load may need to be progressively graduated as the strength of the healing fracture improves. The optimal regime, therefore, may be one of a short initial period of immobilisation followed by graduated interfragmentary micromovement applied to the fracture as it progresses through the stages of healing.

The optimum number of compressive loads and the duration and frequency of treatment sessions needed to enhance fracture healing is also unknown and may in fact vary with each individual fracture. Goodship and Kenwright, ${ }^{4}$ claimed that significant improvement in healing times could be achieved with relatively small amounts of induced micromovement at a fracture site. Rubin and Lanyon, ${ }^{3}$ showed that only 36 cycles at a frequency of $0.5 \mathrm{~Hz}$ per day were required to cause substantial new-bone formation in normal bones. The loads used in the above study were approximately one-third of the normal physiological strains experienced by those bones. The cyclic loading regime used in this current study was not dissimilar to those mentioned above with the loading commencing one week after fracture and progressively increased by $2 \mathrm{~N}$ each week. ${ }^{7}$

The results from the ultrasonography showed that there was no significant difference between the amounts of muscle wasting in the treated group compared with the control group. This was the case at both four and six weeks. Therefore, the action of the inflatable cuff around the muscle does not appear to have any detrimental effect on muscle tissue. Because the action of the cuff relies on the underlying muscle bulk it was also a concern that the uneven distribution of the forelimb flexor and extensor muscle bulk may have caused excessive angulation or translation of the treated fractures. The results obtained from the analysis of the radiographs showed there were no significant differences in the degree of angulation, or the amount of translation, between the treated fractures and the control fractures. Although this study used an osteotomy to simulate the process of fracture healing and extrapolation of the ovine model to the human population has limitations, the results lend support to trialing this cyclic loading method in a patient. The results of this study suggests that, upon receiving the cyclic loading treatment, a plaster cast could be removed at four weeks knowing that the strength of the underlying fracture would not be significantly different from a fracture managed in plaster for six weeks without any loading treatment.

This research was financially supported by The Prince Charles Research Foundation, The Prince Charles Hospital, Rode Rd, Chermside, Brisbane, Australia.

No benefits in any form have been received or will be received from a commercial party related directly or indirectly to the subject of this article.

\section{References}

1. Goodship A, Lanyon LE, McFie H. Functional adaptation of bone to increased stress: an experimental study. J Bone Joint Surg [Am] 1979;61-A:539-46.

2. Panjabi M, White A III, Wolf $\mathbf{J}$ Jr. A biomechanical comparison of the effects of constant and cyclic compression on fracture healing in rabbit long bones. Acta Orthop Scand 1979:50:653-61

3. Rubin C, Lanyon L. Regulation of bone formation by applied dynamic loads. J Bone Joint Surg [Am] 1984;66-A:397-402.

4. Goodship A, Kenwright J. The influence of induced micromovement upon the healing of experimental tibial fractures. J Bone Joint Surg [Br] 1985;67-B:650-5.

5. Kershaw C, Cunningham J, Kenwright J. Tibial external fixation, weight-hearing and fracture movement. Clin Orthop 1993;293:28-36.

6. Park S, O'Connor K, McKellop H, Sarmiento A. The influence of active shear or compressive motion on fracture healing. J Bone Joint Surg [Am] 1998;80-A:868-78.

7. Challis $\mathbf{M}$, Welsh M, Jull G, Crawford R. The effect of intermittent pneumatic soft-tissue compression on simulated distal radial fractures. Clin Orthop 2005;433: 183-8.

8. Rubin C, McLeod K. Promotion of bony ingrowth by frequency-specific, low-amplitude mechanical strain. Clin Orthop 1994;298:165-74.

9. Matsushita T, Kurokawa T. Comparison of cyclic compression, cyclic distraction and rigid fixation: bone healing in rabbits. Acta Orthop Scand 1998;69:95-8.

10. Wolf S, Janousek A, Pfeil J, et al. The effects of external mechanical stimulation on healing diaphyseal osteotomies fixed by flexible external fixation. Clin Biomech 1998;13:359-64

11. Hente R, Cordey J, Rahn B, et al. Fracture healing of the sheep tibia treated using a unilateral external fixator: comparison of static and dynamic fixation. Injury 1999; 30(Suppl 1):44-51.

12. Kenwright $\mathbf{J}$, Goodship A. Controlled mechanical stimulation in the treatment of tibial fractures. Clin Orthop 1989;241:36-47.

13. Kenwright J, Richardson J, Cunningham J, et al. Axial movement and tibial fractures: a controlled randomised trial of treatment. J Bone Joint Surg [Br] 1991;73-B: 654-9.

14. Claes L, Wilke H, Augat P, Rubenacker S, Margevicius KJ. Effect of dynamisation on gap healing of diaphyseal fractures under external fixation. Clin Biomech 1995; 10:227-34

15. Foxworthy M, Pringle R. Dynamization timing and its effect on bone healing when using the Orthofix Dynamic Axial Fixator. Injury 1995;26:117-19.

16. Klein P, Schell H, Streitparth F, et al. The initial phase of fracture healing is specifically sensitive to mechanical conditions. J Orthop Res 2003;21:662-9.

17. Richardson JB, Cunningham JL, Goodship AE, O'Connor BT, Kenwright J. Measuring stiffness can define healing of tibial fractures. J Bone Joint Surg [Br] 1994; 76-B:398-94.

18. Yamada H. Strength of biological materials. Baltimore: Williams \& Wilkins Co., 1970

19. Wolf J Jr, White A III, Panjabi M, Southwick W. Comparison of cyclic loading versus constant compression in the treatment of long-bone fractures in rabbits. $J$ Bone Joint Surg [Am] 1981;63-A:805-10.

20. Egger E, Gottsauner-Wolf F, Palmer J, Arott T, Chau EY. Effective of axial dynamization on bone healing. J Trauma 1993;34:185-92.

21. Goodship A, Watkins PE, Rigby HS, Kenwright J. The role of fixator frame stiffness in the control of fracture healing: an experimental study. J Biomech 1993;26:1027-35.

22. O'Sullivan M, Bronk J, Chao E, Kelly P. Experimental study of the effect of weight bearing on fracture healing in the canine tibia. Clin Orthop 1994;302:273-83.

23. Larsson S, Kim W, Caja V, et al. Effect of early axial dynamization in tibial bone healing: a study in dogs. Clin Orthop 2001;388:240-51.

24. Kenwright J, Gardner T. Mechanical influences on tibial fracture healing. Clin Orthop 1998:355(Suppl):179-90. 